\title{
MV-Algebraic Möbius Transform on the Vietoris Space
}

\author{
Tomáš Kroupa* \\ Institute of Information Theory and Automation of the ASCR \\ Pod Vodárenskou věží 4, 18208 Prague, Czech Republic \\ kroupa@utia.cas.cz
}

Let $X$ be a nonempty finite set and $v$ be a mapping $2^{X} \rightarrow \mathbb{R}$ such that $v(\emptyset)=0$. The Möbius transform [8] of $v$ is the only solution

$$
\mathbf{m}: 2^{X} \rightarrow \mathbb{R}
$$

of the equation

$$
v(A)=\sum_{B \subseteq A} \mathbf{m}(B), \quad \text { for each } A \in 2^{X} .
$$

We will denote the Möbius transform of $v$ by $\mathbf{m}_{v}$. The function $\mathbf{m}_{v}$ can be directly recovered from $v$ as

$$
\mathbf{m}_{v}(A)=\sum_{B \subseteq A}(-1)^{|A \backslash B|} v(B), \quad \text { for each } A \in 2^{X} .
$$

Conversely, let $\mathbf{m}: 2^{X} \rightarrow \mathbb{R}$ be such that $\mathbf{m}(\emptyset)=0$. Put

$$
v_{\mathbf{m}}(A)=\sum_{B \subseteq A} \mathbf{m}(B), \quad \text { for each } A \in 2^{X} .
$$

Then it follows that $\mathbf{m}_{v_{\mathbf{m}}}=\mathbf{m}$.

Our main goal is to find an analogue of the Möbius transform on MV-algebras 4. To this end, replace the boolean algebra $2^{X}$ with a semisimple MV-algebra $M_{X}$. There is no loss of generality in assuming that $X$ is a compact Hausdorff space and $M_{X}$ is a separating MV-algebra of continuous functions over $X$. Since every Möbius transform $\mathbf{m}_{v}$ corresponds to some finitelyadditive measure on $2^{2^{X}}$, the many-valued counterpart of $\mathbf{m}_{v}$ will be an MV-algebraic measure or state [7, 1. Several problems arise in passing to a generalization of the combinatorial Möbius transform. How to replace the mapping

$$
A \in 2^{X} \mapsto\left\{B \in 2^{X} \mid B \subseteq A\right\} \in 2^{2^{X}}
$$

with a map between a pair of MV-algebras? Most importantly, what is the MV-algebra on which a generalized Möbius transform lives?

We start with the construction of an MV-analogue of $2^{2^{X}}$. Observe that (3) can be viewed as a mapping sending each $A \in 2^{X}$ to the set of principal filters to which $A$ belongs. Due to the Galois connection between closed subsets of $X$ and the filters in the MV-algebra $M_{X}$ that are intersections of maximal filters, we may replace the family of such filters with $\mathcal{K}_{X}$, the set of all closed subsets of the compact Hausdorff space $X$. In the following we always assume that the hyperspace $\mathcal{K}_{X}$ is endowed with the topology whose subbasis is formed by the following sets:

$$
\begin{aligned}
& A^{+}=\left\{B \in \mathcal{K}_{X} \mid B \subseteq A\right\}, \\
& A^{-}=\left\{B \in \mathcal{K}_{X} \mid B \cap A \neq \emptyset\right\},
\end{aligned}
$$

*The work of the author was supported by the grant GAČR 13-20012S. 
where $A$ ranges in the open subsets of $X$. The resulting topology on $\mathcal{K}_{X}$ is called the Vietoris topology and the space $\mathcal{K}_{X}$ is the Vietoris space of $X$. See [2, Section 2.2] or [5, Section III 4] for the basic properties of the Vietoris topology and other hyperspace topologies.

Proposition 1. Let $X$ be a compact Hausdorff space.

(i) The Vietoris space $\mathcal{K}_{X}$ is compact and Hausdorff.

(ii) If $X$ is metrizable by a metric d, then the Vietoris topology coincides with the Hausdorff metric topology on $\mathcal{K}_{X}$ induced by $d$.

(iii) The mapping $x \mapsto\{x\}$ is a homeomorphism of $X$ and the space $\{\{x\} \mid x \in X\}$ considered in its subspace Vietoris topology.

(iv) Let $g: X \rightarrow Y$ be a continuous mapping into a compact Hausdorff space $Y$. Then the mapping $\hat{g}: \mathcal{K}_{X} \rightarrow \mathcal{K}_{Y}$ sending each $A \in \mathcal{K}_{X}$ to its image under $g$ is continuous for the Vietoris topologies.

In this contribution we generalize some results from [6], where only the case of secondcountable (and thus metrizable) space $X$ is considered. The property (ii) thus guarantees that this is an extension of our previous approach. First, we extend (3) to MV-algebras. Consider the mapping

$$
\rho_{a}(B)=\left\{\begin{array}{ll}
\min \{a(x) \mid x \in B\} & \emptyset \neq B \in \mathcal{K}_{X}, \\
1 & B=\emptyset,
\end{array} \quad a \in M_{X} .\right.
$$

Loosely speaking, the number $\rho_{a}(B)$ determines a degree to which $a$ belongs to the filter generated by the closed set $B$.

Proposition 2. Let $a \in M_{X}$. The mapping

$$
\rho_{a}: \mathcal{K}_{X} \rightarrow[0,1]
$$

sending each $B \in \mathcal{K}_{X}$ to $\rho_{a}(B)$ is continuous in the Vietoris topology.

Proof. We write $\rho_{a}=\min \circ \hat{a}$, where $\hat{a}$ is as in Proposition 1 (iv) and

$$
\min : \mathcal{K}_{[0,1]} \rightarrow[0,1]
$$

takes $B \in \mathcal{K}_{[0,1]}$ to its minimal element, where the hyperspace $\mathcal{K}_{[0,1]}$ is endowed with the Vietoris topology and $[0,1]$ has the usual Euclidean topology. Since $\hat{a}$ is continuous, we need only show that min is continuous. This is carried out routinely by checking that, for each $b, c \in[0,1]$, the pre-images of subbasic open sets $[0, b)$ and $(c, 1]$ under min are subbasic opens in $\mathcal{K}_{[0,1]}$.

Let $C\left(\mathcal{K}_{X}\right)$ be the MV-algebra of all continuous functions $\mathcal{K}_{X} \rightarrow[0,1]$.

Proposition 3. The mapping $\rho: a \in M_{X} \mapsto \rho_{a} \in C\left(\mathcal{K}_{X}\right)$ has the following properties:

(i) $\rho_{0}=0, \rho(1)=1$;

(ii) $\rho$ is injective;

(iii) $\rho_{a \oplus b} \geq \rho_{a} \oplus \rho_{b}$;

(iv) if $a_{1}, \ldots, a_{n} \in M_{X}$, then $\rho_{\bigwedge_{i=1}^{n} a_{i}}=\bigwedge_{i=1}^{n} \rho_{a_{i}}$. 
Thus $\rho$ is an embedding of the meet-semilattice reduct $\left\langle M_{X}, \wedge, 1\right\rangle$ of $M_{X}$ into the meetsemilattice $\left\langle C\left(\mathcal{K}_{X}\right), \wedge, 1\right\rangle$. Let $\rho_{M_{X}}$ be the corresponding image of $M_{X}$. Instead of the whole $\mathrm{MV}$-algebra $C\left(\mathcal{K}_{X}\right)$, we can use the MV-algebra generated by the meet-semilattice $\left\langle\rho_{M_{X}}, \wedge, 1\right\rangle$, but its structure does not seem more clear. There is, however, a direct description of each element of $\rho_{M_{X}}$ based on the values in its range. Let $a \in M_{X}$ and $y \in \hat{a}(X)$. Then

$$
\rho_{a}^{-1}(y)=\left\{B \in \mathcal{K}_{X} \backslash\{\emptyset\} \mid \hat{a}(B) \subseteq[y, 1], y \in \hat{a}(B)\right\} .
$$

It is easy to see that $\rho_{a}^{-1}(y)$ is a lattice of sets, which is a closed subset of $\mathcal{K}_{X}$. Hence each function $\rho_{a}$ attains a constant value over some sublattice of $\mathcal{K}_{X}$.

Since the MV-algebra $C\left(\mathcal{K}_{X}\right)$ is our analogue of the domain for the Möbius transform, which can be identified with a finitely-additive measure, we will consider as a plausible candidate for a generalized Möbius transform any bounded measure $\mathbf{m}: C\left(\mathcal{K}_{X}^{\prime}\right) \rightarrow \mathbb{R}$ in the sense of [1], where $\mathcal{K}_{X}^{\prime}=\mathcal{K}_{X} \backslash\{\emptyset\}$. Then, observing the identity [2], put

$$
v_{\mathbf{m}}(a)=\mathbf{m}\left(\rho_{a}\right), \quad a \in M_{X} .
$$

As proved in [6] the function $v_{\mathbf{m}}$ has the following form:

$$
v_{\mathbf{m}}(a)=\int_{0}^{1}\left(\beta_{1}-\beta_{2}\right)\left(a^{-1}([t, 1])\right) \mathrm{d} t,
$$

where each $\beta_{i}: \mathcal{K}_{X} \rightarrow \mathbb{R}$ is the so-called totally monotone capacity [3].

Theorem 1 ([] $]$ ). Let $v: M_{X} \rightarrow \mathbb{R}$ be such that

$$
v(a)=\int_{0}^{1}\left(\beta_{1}-\beta_{2}\right)\left(a^{-1}([t, 1])\right) \mathrm{d} t,
$$

for some totally monotone capacities $\beta_{i}$. Then there exists a unique bounded measure $\mathbf{m}_{v}$ on $C\left(\mathcal{K}_{X}^{\prime}\right)$ such that $v(a)=\mathbf{m}_{v}\left(\rho_{a}\right)$, for every $a \in M_{X}$. Moreover, we have $\mathbf{m}_{v_{\mathbf{m}}}=\mathbf{m}$, for every bounded measure $\mathbf{m}$ on $C\left(\mathcal{K}_{X}^{\prime}\right)$.

The previous theorem singles out the class of functions $v: M_{X} \rightarrow \mathbb{R}$ having a generalized Möbius transform: they are exactly those functions $v$ fulfilling the equation (5) for some Choquet capacities $\beta_{1}$ and $\beta_{2}$. In particular, if $v$ has the generalized Möbius transform, then $v$ must be a difference of two totally monotone functions on $M_{X}$. Although we were not able to describe the class of such functions in purely algebraic terms, we can identify some of its important members. The following functions possess the generalized Möbius transform:

(i) Each homomorphism of $M_{X}$ into the standard MV-algebra $[0,1]$.

(ii) Every bounded measure and, a fortiori, every state on $M_{X}$.

(iii) Every function $v_{B}(a)=\min \{a(x) \mid x \in B\}$, where $B \in \mathcal{K}_{X}^{\prime}$.

(iv) Each convex combination of functions from (iii) and their pointwise limits.

\section{References}

[1] G. Barbieri and H. Weber. Measures on clans and on MV-algebras. In Handbook of Measure Theory, Vol. I, II, pages 911-945. North-Holland, Amsterdam, 2002. 
[2] G. Beer. Topologies on closed and closed convex sets, volume 268 of Mathematics and its Applications. Kluwer Academic Publishers Group, Dordrecht, 1993.

[3] G. Choquet. Theory of capacities. Ann. Inst. Fourier, Grenoble, 5:131-295 (1955), 1953-1954.

[4] R. L. O. Cignoli, I. M. L. D'Ottaviano, and D. Mundici. Algebraic foundations of many-valued reasoning, volume 7 of Trends in Logic-Studia Logica Library. Kluwer Academic Publishers, Dordrecht, 2000.

[5] P. T. Johnstone. Stone spaces. Cambridge University Press, 1982.

[6] T. Kroupa. Generalized Möbius transform of games on MV-algebras and its application to Cimminotype algorithm for the core. In Optimization Theory and Related Topics, volume 568 of Contemporary Mathematics, pages 139-158. Amer. Math. Soc., Providence, RI, 2012.

[7] D. Mundici. Averaging the truth-value in Lukasiewicz logic. Studia Logica, 55(1):113-127, 1995.

[8] G.-C. Rota. On the foundations of combinatorial theory. I. Theory of Möbius functions. Zeitschrift für Wahrscheinlichkeitstheorie und Verwandte Gebiete, 2:340-368 (1964), 1964. 